PACS 73.40.-c, 85.35.Be

\title{
Model of heterotransistor with quantum dots
}

\author{
V.I. Timofeyev, E.M. Faleyeva \\ National Technical University of Ukraine "KPI" \\ 37, prospect Peremogy, 03056 Kyiv, Ukraine \\ E-mail:v.timofeyev@kpi.ua; elena.faleeva@gmail.com
}

\begin{abstract}
Heterostructure transistors with quantum dots (QD) are now very perspective devices because of their higher velocities of electrons in the channel. Simulation results for concentration and carrier velocity distributions depending on the QD size, concentration and location were presented in this paper. It is shown that presence of QD in the channel causes a significant increase of current. Also, QD location and concentration influence to the output characteristics of transistor was established.
\end{abstract}

Keywords: heterostructure transistor, quantum dot.

Manuscript received 10.02.10; accepted for publication 25.03.10; published online 30.04.10.

\section{Introduction}

Heterotransistors with quantum dots (QD) are very perspective devices because of the increased electron drift velocity in the transistor channel, as it was shown in the previous work [1]. That's why, simulation of physical processes in these structures is an actual problem. Pure analytical models can not be used to such simulations, and implementation of numerical methods is needed for solving the relaxation equations. The solution must take into account heterogeneities that make structure's properties special. In this case, these specialties are the processes in heterojunction and quantum dot system. The object of this paper is to analyze the simulation results for heterotransistors with $\mathrm{QD}$, continuing the authors' previous works $[2,3]$.

\section{Simulation}

We obtained the results of modeling by solving the system of relaxation equations [3] for the heterostructure transistor with QD. Both quantum wells and QD were built into the potential distribution as potential stairs regardless size quantization effects. To take into account the quantum effects, the same system of equations was solved, where the electron distribution in the quantum well were found from a simultaneous solution of the Poisson equation (1) and the stationary Schrödinger equation (2):

$$
\begin{aligned}
& \Delta \varphi(x, y)=-\frac{\rho}{\varepsilon \varepsilon_{0}}, \\
& -\frac{\hbar^{2}}{2 m^{*}} \Delta \psi(x, y)+\left(\varphi(x, y)-E_{0}\right) \psi(x, y)=0 .
\end{aligned}
$$

The main energy level $E_{0}$ was found from the known expression, and the concentration profile is found according to the Fermi-Dirac statistics [4].

\section{Simulation results}

Presented in Figs $1 \mathrm{a}$ and $1 \mathrm{~b}$ are the carrier concentration distributions for the heterotransistors with QD and the gate width $0.25 \mu \mathrm{m}$, and with the interelectrode distances of 0.85 and $0.35 \mu \mathrm{m}$, respectively.

With channel length shortening (Fig. 1b) the "ballistic" pattern of the carrier transport is more valuable. Thus, the concentration increase is observed near the drain only, because of intervalley transitions and electron heating.

During the self-organization process, QD can be situated non-uniformly in the channel layer. That's why, these circumstances should be considered when researching and analyzing reproducibility of QD heterotransistor characteristics. In Figs $2 a$ and $2 b$, the mobile carrier concentration distributions are shown for the random QD localization in the channel area, and in Figs $3 \mathrm{a}$ and $3 \mathrm{~b}$ carrier velocity distributions are shown. 
These distributions were found for the same concentration of QD and their regular position near the drain and source, respectively.

Random QD location in the transistor cannel leads to local heterogeneities in the concentration, electrical field and mean values of velocity distributions. Distributions of physical characteristics may be quite different for two cases of random QD locations. This fact verifies that transistor conductivity depends not only on QD in the channel but also on the order of QD location in this channel.

Also for these models, there is a big interest to QD size influence on transistor characteristics. As expected, embedding QD to the channel increases the transistor current. The bigger is the QD diameter, the larger is the contribution to the carrier concentration from each QD emitting electrons. Shown in Fig. $4 \mathrm{a}$ is the distribution for electrons in the channel for QD foundation area close to $1 \mu \mathrm{m}^{2}$. These QD are located between the gate and source of the transistor. It's interesting that the carrier velocity in the transistor stays almost constant (Fig. 4b).

Current-voltage characteristics were calculated for this structure (Fig. 5, solid lines).

Calculation of the current was made with account of the carrier velocity and concentration with the assumption that all the QD are ionized, which is completely fair, as all the QD are located in the area of high electric fields.

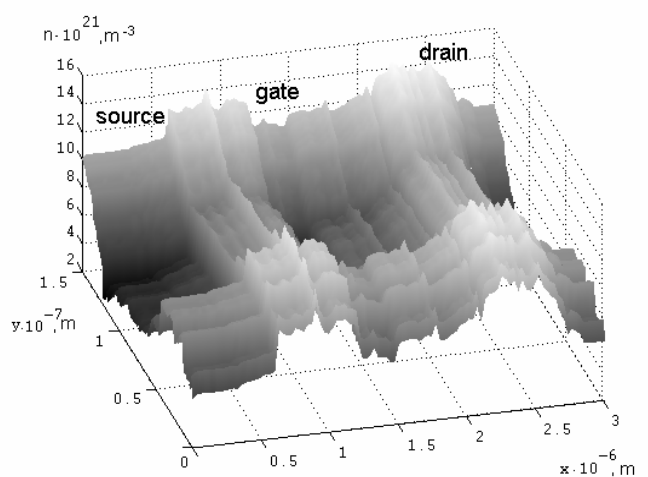

a)

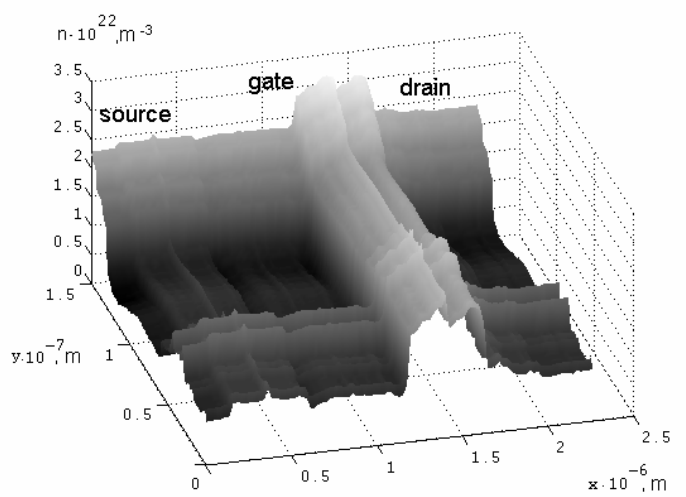

b)

Fig. 1. Carrier concentration distribution for heterotransistors with QD and gate width $0.25 \mu \mathrm{m}$ as well as the interelectrode distances of 0.85 (a) and $0.35 \mu \mathrm{m}(\mathrm{b})$.

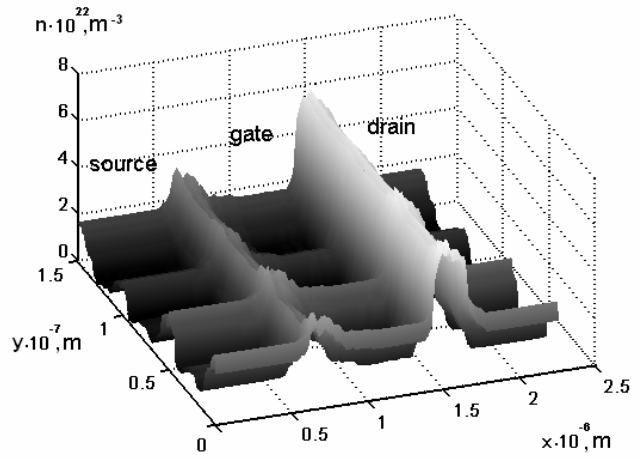

a)

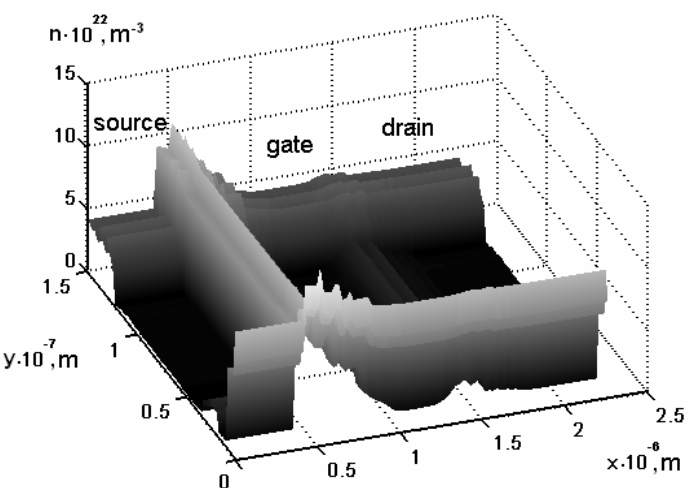

b)

Fig. 2. Distributions of the carrier concentration for the same QD concentration, but with random positions of QD. QD concentration is about $10^{12} \mathrm{~cm}^{-2}$.

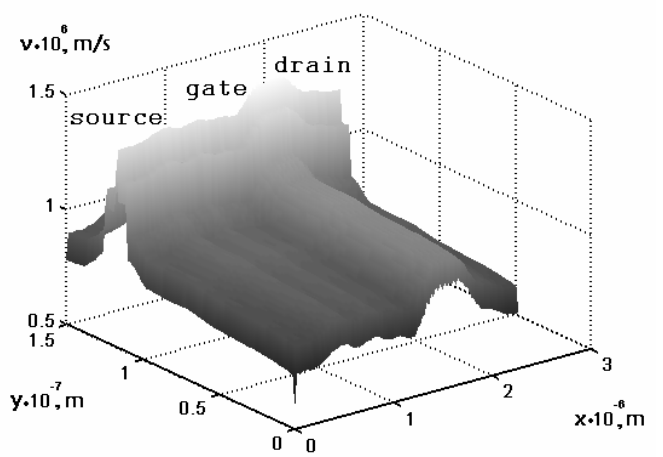

a)

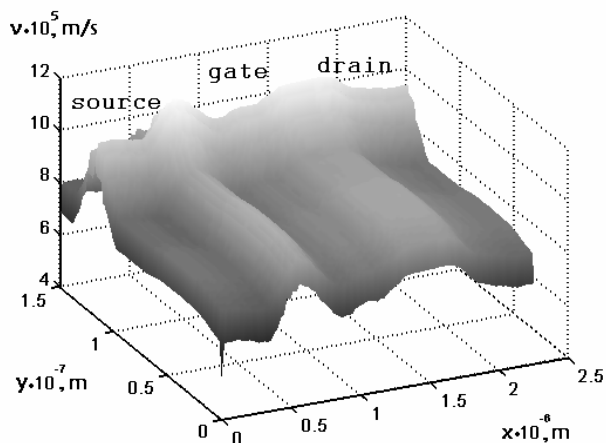

b)

Fig. 3. Distribution for mean values of carrier velocity for heterotransistor with QD, when QD locate near drain (a) and source (b) gate boundaries. 


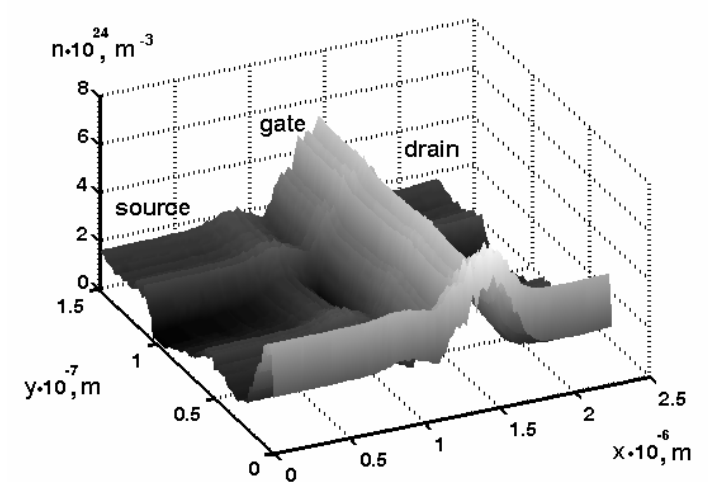

a)

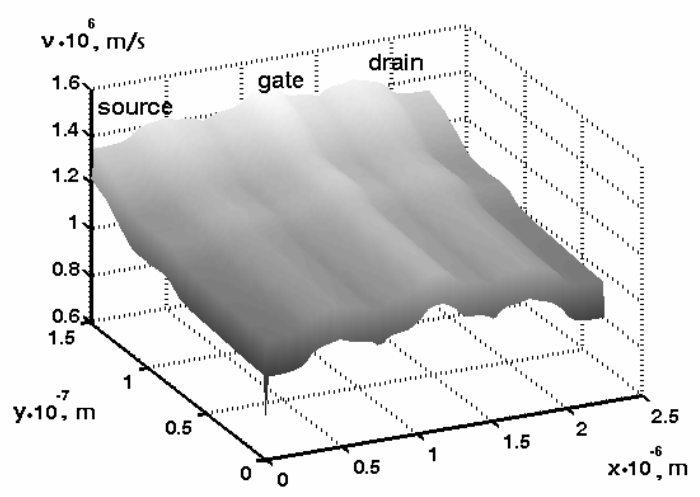

b)

Fig. 4. Carrier concentration (a) and velocity (b) distributions for QD area with the square about $1 \mu \mathrm{m}^{2}$.

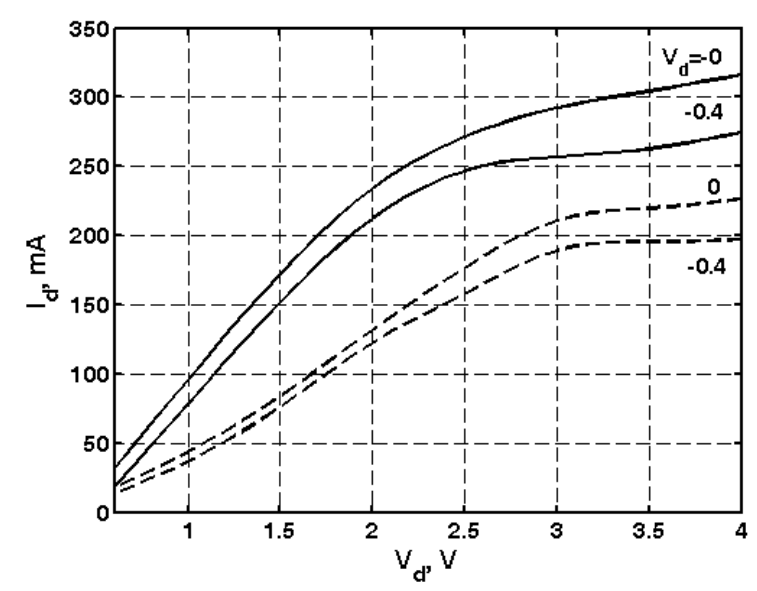

Fig. 5. I-V characteristics of heterotransistor with QD. Solid lines - heterotransistor with QD, dotted lines - heterotransistor without QD.
The distance between QD and heterojunction as well as their sizes do not meet the conditions of polar optical phonons quantization and prevail influence. Thus, all the scattering mechanisms for the submicron structures were considered in the current computation.

\section{Conclusions}

1. In a heterotransistor with $\mathrm{QD}$, the channel length decrease changes the carrier transport character dramatically. The space-charge region near the sourcegate boundary (Fig. 1a) is absent in the case of Fig. 1b.

2. No regularities for the obtained carrier concentration distributions and output current values were observed for random QD localization in the channel.

3. Velocity values for heterotransistor with QD, when QD are located near a drain boundary of the gate (Fig. 3a), are 1.5 times higher than in a heterotransistor with QD near source boundary of the gate (Fig. 3b).

4. The size of quantum dots influences on the carrier concentration and velocity. It is demonstrated in Figs 1b and 4a. QD sizes ratio for these structures is 1:4.

QD embedding to the channel of heterotransistor leads to an evident increase of the output current. The definite value of this current is a function of size, concentration and location of QD relatively to the channel.

\section{References}

1. Yu.K. Pozhela, V.G. Mokerov, Big increasing of maximum drift velocity in the channel of field-effect heterotransistor // Fizika tekhnika poluprovodnikov 40(3), p. 362-366 (2006), in Russian.

2. V.I. Timofeyev, E.M. Faleyeva, Analysis of processes in submicron heterotransistor with quantum dots // Electronics and communications, Subject publication "Problems of electronics", p. 100-103 (2008).

3. V.I. Timofeyev, E.M. Faleyeva, Methods for numerical solution of relaxation equations system for submicron heterostructures // Electronics and communications 6, p. 5-9 (2008).

4. V.I. Zubkov, Simulation of capacitance-voltage characteristics of heterostructures with quantum wells using self-consistent solution of Schrödinger's and Poisson's equations // Fizika tekhnika poluprovodnikov 40(10), p. 1236-1240 (2006), in Russian. 